\title{
From DNA to form: the achaete-scute complex
}

\author{
Alain Ghysen and Christine Dambly-Chaudière \\ Laboratory of Genetics, Université de Bruxelles, 1640 Rhode-St-Genèse, Belgium
}

The large bristles of flies (macrochaetes) are sense organs that form at precise locations on the thorax and the head, so much so that each has been given a name. The first scute mutant, a fly that lacked a few bristles, was discovered in 1916. As more mutations were found, it was observed that different alleles removed different subsets of bristles. A few of the new mutations affected a subset of bristles totally different from the subset eliminated by the original scute mutation, $s c^{1}$. Such mutations fully complement $s c^{1}$ and therefore were assumed to define a new gene named achaete.

Early investigators were struck by the fact that each scute allele removed a specific subset of bristles, and they set out to understand whether and how the structure of the gene could explain such specificity. One theory, originating with a group of Russian genetists (who isolated many scute mutations in the late 1920s, after Müller discovered that X-rays are mutagenic) held that scute contained distinct subgenes, each responsible for the development of one or a few bristles (Serebrowsky and Dubinin 1930; Agol 1931; Dubinin 1932, 1933). However, western geneticists asserted that the specificity was not in scute, but resulted from regional differences in the epidermis and/or involved the genetic system as a whole (Goldschmidt 1931, 1938; Sturtevant and Schultz 1931; Child 1935).

At the heart of the disagreement lay the problem of the divisibility of the gene. This was understood clearly by $\mathrm{H}$. Müller, who had worked with both sides. He demonstrated that the chromosome segment affected by scute mutations contains at least three adjacent genes, two of which (achaete and scute) are essential for the formation of bristles, while the third (lethal of scute) is essential for viability (Müller and Prokofyeva 1935). Yet, in conclusion to an outstanding piece of work that involved the feat of planning and bringing to fruition 29 consecutive crosses, he admitted that there is no way to decide. whether this region may be further subdivided into elements that specify individual bristles, or whether the origin of the specificity must be found elsewhere (Raffel and Müller 1940). Thus, a deadlock had been reached in 1940, with no prospect of a solution. Yet, as we shall see, it now appears that both theories contain an element of truth and reflect two different aspects of a finely engineered piece of genetic machinery.

We dedicate this review to Prof. Jean Brachet, in admiration.
The modern history of scute began in 1975, when A. Garcia-Bellido (then visiting E.B. Lewis, the father of another famed complex) set out to analyze scute both genetically and developmentally. This dual approach was imposed by his conviction that the genetic organization of this segment of chromosome and its developmental function are linked intimately and therefore must be addressed simultaneously if any progress were to be made (Garcia-Bellido 1979, 1981).

The functional analysis (Garcia-Bellido and Santamaria 1978) revealed that there is more in scute than meets the eye: Not only does a deletion of the achaetescute segment remove all bristles, but it also eliminates other types of sense organs (as well as some central neurons, an aspect that will not be considered here). Furthermore, this analysis suggested that achaete and scute are involved in the initial decision to differentiate a bristle, and not in the differentiation process itself--a conclusion amply supported by subsequent genetic and molecular work on overexpressing alleles that develop supernumerary bristles (Campuzano et al. 1986; GarciaAlonso and Garcia-Bellido 1986).

The genetic analysis (Garcia-Bellido 1979) confirmed and extended that of Müller, resulting in the following picture for the 11 macrochaetes of the thorax. The distalmost gene of the series, achaete (ac), is required for the formation of three macrochaetes (Fig. 1, left panel). Proximal to $a c$ is the gene scute (sc), which is required for the presence of nine macrochaetes (Fig. 1, second panel). The achaete and scute sets of bristles are complementary except for one bristle, which requires both achaete and scute (bristle number 14, Fig. 1, is affected by $a c$ as well as by $s c$ mutations). Proximal to $s c$ is the gene lethal of scute (lsc), which is not involved in bristle development. Finally, a region proximal to $l s c$ is also required for the development of scute bristles (that is, mutations on either side of the gene $1 s c$ affect the same bristles and do not complement each other). This peculiar organization indicates that the genes achaete and scute are part of a gene complex hereafter called Achaete Scute Complex (AS-C).

This genetic dissection of AS-C paved the way for the molecular analysis undertaken in 1980 by J. Modolell and his co-workers (Carramolino et al. 1982). One of the outcomes of the molecular analysis of AS-C is that the phenotype of most scute alleles can now be correlated with their location on the chromosome (Campuzano et al. 1985, Fig. 2). This could not be done before, because 

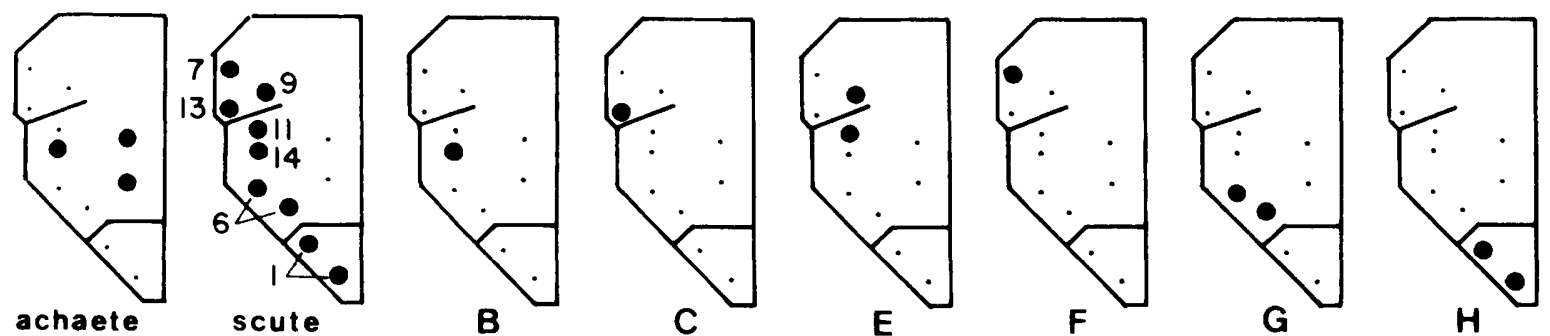

Figure 1. Diagram of the left half of the dorsal mesothorax, showing the location of the 11 precisely located macrochaetes (dots). The achaete and scute sets of bristles are shown on the left (Garcia-Bellido 1979). The numbers are as in Figure 2. The scute set of bristles can be subdivided as shown in the right panels, according to which region of the AS-C complex must be present in addition to the T4 coding sequence for the development of that or those particular bristles.

the incidence of recombination in this region is too low to permit the meiotic mapping of alleles within AS-C. Furthermore, several transcripts were also identified, two of which (T4 and T5) correspond, respectively, to the scute and achaete genes. A somewhat surprising fact that emerged from this molecular mapping is that all scute mutations map downstream of $\mathrm{T} 4$, the scute transcript, except for two alleles, $s c^{8}$ and $s c^{v 2}$, which have such a weak and erratic phenotype that one wonders how they were picked up in the first place. Some alleles map quite far from the transcribed region; for example, $s c^{19}$ is a breakpoint more than $40 \mathrm{~kb}$ away, with several other transcribed regions in between. It also appears that the closer a mutation is to $\mathrm{T} 4$, the more extreme is its phenotype. At first sight this progressive effect fits with the idea that the specificity shown by different scute alleles reflects local differences in threshold or sensitivity in the developing adult epidermis. Thus, only the most sensitive sites would be affected by the weakest (furthest from $\mathrm{T} 4$ ) alleles, while the least sensitive bristles would be removed only by the most extreme (closest to T4) alleles. However, a quite different interpretation can be proposed, as we shall see.

\section{The 1987 harvest}

The dual analysis summarized above led to the realization that the AS-C genes may be a key to understanding how a sequence of DNA encodes for a two-dimensional pattern, and it provides the conceptual frame in which the 1987 papers will now be analyzed.

The first paper (Dambly-Chaudière and Ghysen 1987) deals with the effect of AS-C in the larva, taking advantage of the fact that the sensory system of the larva includes several types of sense organs arranged in different patterns in the various body segments /Campos-Ortega and Hartenstein 1985; Dambly-Chaudière and Ghysen 1986): most of the previous work on AS-C had been restricted to one type of sense organs (the bristles) in part of one segment (the dorsal mesothorax). This study concluded that AS-C defines a 'primary' pattern which is segmentally repeated, and that the segmental differences in the final pattern result from subsequent modifications of this early pattern. The primary pattern itself results from the superimposition of two subpatterns, A and $B+C$, each of which contains representatives of the different types of sensilla present in the larva (monoinnervated hairs and papilla, multiinnervated pegs). Subpattern A is formed whenever achaete or scute is present. Thus, while achaete and scute define two complementary subsets of adult bristles, they both define the same subset of larval sense organs, and can actually replace each other in the larva. Subpattern B + C depends on a new gene located in the proximal-most region of the complex. We propose to call this new gene asense (ase) since its removal causes a lack of larval sense organs. The absence of asense has little if any effect on the adult bristles.

Two other papers report the spatial distribution of three of the AS-C transcripts in the embryo (Cabrera et al. 1987; Romani et al. 1987). These analyses show that AS-C transcripts accumulate in the regions where neuroblasts will later segregate from the epidermis; however, transcripts are not detected in differentiating neurons, confirming that AS-C is involved in the initial decision and not in the differentiation. The distributions of $\mathrm{T} 4$ and $\mathrm{T} 5$ are largely overlapping, in agreement with the conclusion that achaete and scute can substitute for each other in the determination of larval sense organs. Finally, in at least one case the transcript is first distributed in seven stripes, suggesting that the early control of AS-C expression may depend directly on some of the pair-rule segmentation genes.

A fourth paper reports sequence data that show that T4 and T5 share extensive sequence homologies (Villares and Cabrera 1987), a good indication that different AS-C products perform basically similar functions. This homology extends to $\mathrm{T} 3$, a transcript corresponding to the lethal of scute function involved in the determination of central neurons. A search based on this homology allowed the detection of at least one additional homologous transcript that is coded for by the proximal-most region of AS-C and presumably corresponds to the newly recognized asense gene (initially two homologous transcripts were described in this region; however, recent data from Modolell's group suggest that there is only one, as shown in Fig. 2).

It must be said here that the association of particular phenotypes to identified coding sequences remains somewhat tentative, due to the lack of point mutations 
in the complex. All but one of the mutations in AS-C analyzed so far are breakpoints and insertions outside of coding sequences, or deletions that may remove 0,1 , or more coding sequences. The one point mutation, a nonsense mutation in the $\mathrm{T} 4$ coding region /Villares and Cabrera 1987), occurred as a second mutation on an $a c^{3}$ chromosome and has not been separated from the $a c^{3}$ breakpoint (the double mutant lacks all thoracic bristles). Thus, the assignment presented in the upper part of Figure 2 is not demonstrated, even though it is very likely correct.

A final observation derived from the sequence data of Villares and Cabrera is the existence of a substantial similarity between the AS-C genes and the oncogene myc. It is worth mentioning in this context that in most arthropods the initial decision of an ectodermal cell to become a sense organ is followed by two differentiative mitoses. In the case of monoinnervated sense organs such as the macrochaetes, the four sib cells differentiate the bristle shaft, its socket, the neuron, and a sheath cell, respectively, (reviewed in Bate 1978; the development of multiinnervated organs involves additional rounds of mitosis). In addition, the earliest indication that a cell has decided to become a macrochaete is that it changes its pattern or mitosis, in contrast with the surrounding cells, which keep dividing regularly until they finally differentiate as epidermis (Garcia-Bellido and Merriam 1971). These observations raise the intriguing possibility that the determining factor for the formation of a sense organ is the commitment to a given pattern of mitosis, and that the function of AS-C genes is to select or impose this particular pattern.

All these results point to a causal role of AS-C genes in the development of the sensory system in both the larva and the adult, and also suggest a very elaborate regulation of the different AS-C genes, both spatially and temporally. The question of how this regulation is achieved becomes all the more crucial then, because the spatial restriction of AS-C expression seems to be the primary factor that determines the basic pattern of sense organ determination.

\section{Position-specific control sites}

A paper by Ruiz-Gomez and Modolell, in the December issue of Genes \& Development, addresses this last question in the case of the macrochaetes of the adult, and proposes a fascinating answer. These authors performed a careful molecular and phenotypic analysis of 74 terminal deficiencies with breakpoints scattered all over the left half of the complex (five of which are shown by arrowheads, Fig. 2), with the aim of assessing 'who does

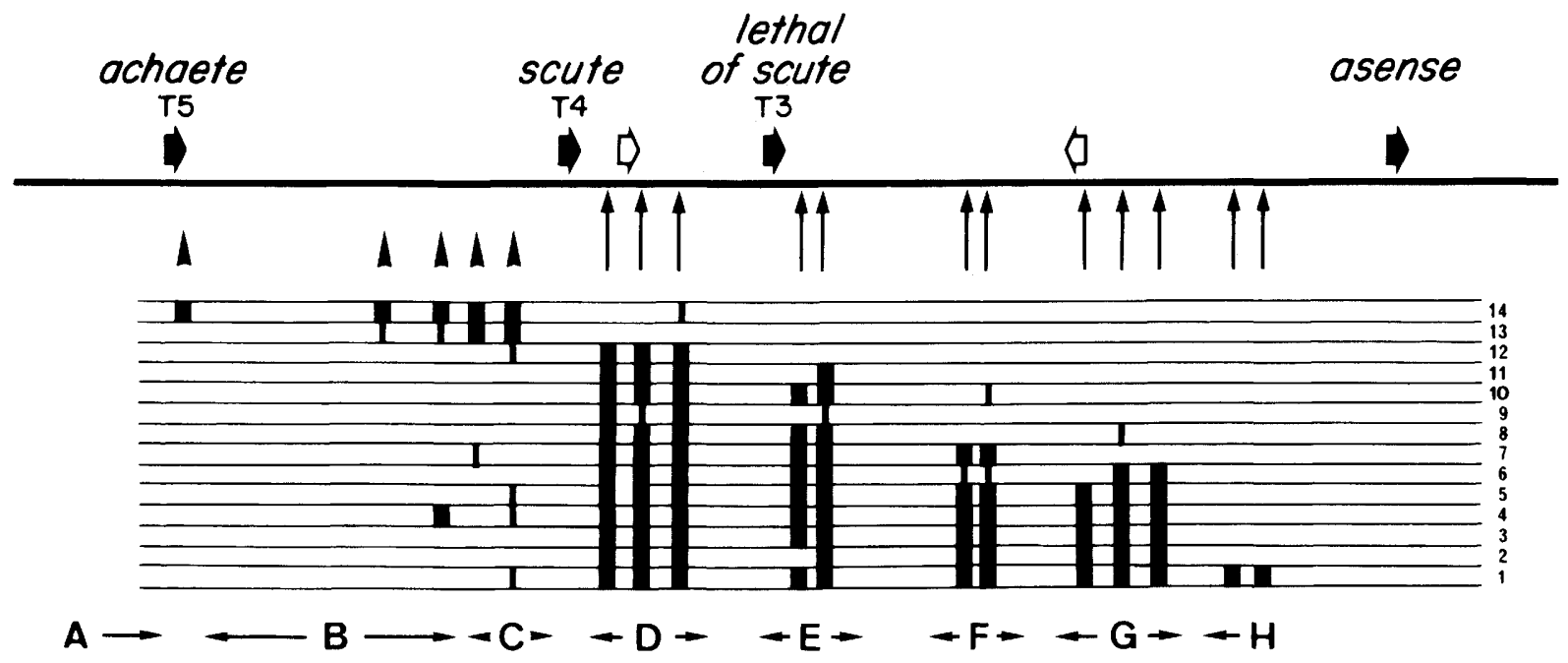

Figure 2. Molecular and phenotypic map of AS-C. The upper line shows the chromosome segment of interest, which is $100 \mathrm{~kb}$ long. Distal is to the left, proximal to the right. The arrows above the line represent the transcripts identified so far, at least four of which share homologous sequences (black arrows). The corresponding genes are achaete (ac), scute (sc), lethal of scute (lsc), and asense (ase, see text). The vertical arrows under the line mark the location of $s c u t e$ breakpoints (inversions, translocations). From right to left: $s c^{19}$, $s c^{260.22}, s c^{29}, s c^{7}, s c^{52}, s c^{9}, s c H, s c^{K A 8}, s c^{260.14}, s c^{4}, s c^{s 1}, s c^{L 8}$. The arrowheads mark the breakpoints of five of the terminal deletions studied by Ruiz-Gomez and Modolell, from right to left, 650, 618, 524, 630, and 212. The lower panel represents the scute phenotype of each mutant. The numbers at the right represent the different scute bristles on the head and notum, as follows: 1 , scutellars; 2 , postvertical; 3, ocellar; 4, sternopleurals; 5, anterior and midorbitals; 6, postalars; 7, anterior notopleural; 8, posterior orbital; 9, presutural; 10, postvertical; 11, anterior supra-alar; 12, humerals; 13, posterior notopleural; 14, posterior supra-alar /the location of the notum bristles are shown Fig. 1). Large bars mean a suppression of more than $50 \%$, light bars, more than $10 \%$. The data on the terminal deletions are from heterozygotes for a deletion of the scute gene, $\operatorname{In}(1) s c 8 L s c^{4 R}$, so that only the $s c u t e$ phenotype is seen. We have illustrated only deletions that are viable over a deletion for the entire complex. At the bottom of the figure, the complex is divided into seven regions, $\mathrm{A}-\mathrm{H}$, according to the variations in phenotype. This figure is a compendium of data and figures provided in Campuzano et al. (1986), Villares and Cabrera (1987), and Ruiz-Gomez and Modolell (1987), and Modolell et al., unpublished. 
what' among the nontranscribed regions. This approach allowed them to define the three regions labeled A, B, and $\mathrm{C}$ in Figure 2.

Deletions that extend into A reduce the level of expression of $\mathrm{T} 5$, the achaete transcript. The effect is almost graded linearly: With very few exceptions, bigger deletions result in lower levels of T5 and a more extensive removal of achaete bristles. Therefore, this stretch of DNA is supposed to provide an appropriate configuration for the synthesis of $\mathrm{T} 5$, but is not responsible for its spatial restriction. Deletions that extend into $B$ remove all the achaete bristles, as expected from the absence of T5. However, even if the T5 coding sequence and its upstream control region A are present on the homologous chromosome, one bristle is still removed (bristle 14 in Fig. 2), suggesting that the development of this bristle depends on region $B$. Deletions that enter $C$ remove a second bristle, bristle 13 in Figure 2, which depends specifically on the scute transcript $\mathrm{T} 4$. The astonishing fact is that these are precisely the two bristles that remain unaffected by virtually all scute mutations located to the right of $\mathrm{T} 4$, even the most extreme ones (leftmost arrows in Fig. 2).

These results cast some doubt on the idea that the specificity of the phenotype is due to differential sensitivities of the different bristles to reduced levels of $\mathrm{T} 4$. Thus, the authors propose a radically different interpretation: The expression of T4 is under the control of cisacting, site-specific elements that respond to topological cues. This would be the explanation for the seriation of phenotypes shown in Figure 2-as breakpoints (or insertions) get closer to T4, they disconnect more and more control sites from the gene.

Do we have to envisage an exquisitely precise mechanism whereby a control site specifically activates scute in the one cell that will become a bristle? Fortunately not. In 1940, Wigglesworth proposed that the regularly spaced pattern of bristles found in many insects could be explained by a simple mechanism of lateral inhibition, i.e., if a cell that has become determined inhibits its neighbors over a certain distance, then no other bristle will form within the radius of inhibition. A similar mechanism may ensure that only one bristle will form even if scute is expressed in a region much larger than a single cell. Assuming the first cell to be determined prevents its fellows from following the same fate, then only one bristle will form within this region. Furthermore, computer simulations have shown that the bristle will form at a reproducible location even if the regional control of AS-C expression is not very precise (Ghysen and Richelle 1979|. Needless to say, this mechanism can also account for the determination of two bristles in the same area, provided the region of AS-C expression is larger than the radius of inhibition.

The idea of a regional control of AS-C expression accounts for the observation that in scute mutants, or in mosaic for scute and normal territories, the development of bristles at appropriate locations is independent of the presence of bristles at the other positions /Child 1935; Ghysen 1980). This idea also fits extremely well with Stern's observation that the determination of a bristle depends on the expression of AS-C not only in the cell to be determined, but also in the surrounding cells that will remain epidermal (Stern 1954a, 1956).

The specificity of the scute control sites hypothesized by Ruiz-Gomez and Modolell can be guessed from the stepwise aggravations of the phenotype, as apparent in Figure 2. Interestingly, each site defines a region of the notum where one or at most two bristles will form (Fig. 1 ), as would be expected if these sites responded to activators present only in some regions of the adult epidermis. A direct test of the idea that scute is indeed expressed in defined regions, each depending on a particular control site, may soon become possible with the help of anti-scute antibodies now being developed in the labs of J. Modolell and of Y.N. and L.Y. Jan.

\section{What activates $A S-C$ ?}

In the embryo, good candidates for regionally localized activators are the segmentation genes (Nusslein-Volhard and Wieschaus 1980; Gergen 1987). The early bisegmental pattern of the T3 transcript, so typical of the early distribution of many of these genes, is certainly suggestive of such a control (Cabrera et al. 1987; Wilkins 1987). But what could be the specific activators in the adult epidermis? At least some of the segmentation genes essential for the antero-posterior organization of the embryo are also required in defined regions of the adult epidermis (see refs. in Perrimon and Mahowald 1987). This raises the truly remarkable possibility that at least some of the genes that may regulate AS-C spatially during early embryogenesis, and thereby define where larval sense organs will develop, are also instrumental in controlling the regional expression that will define where the adult bristles will form in the adult epidermis.

A clear case of a segmentation gene that is also involved in the adult pattern of bristles is hairy $(h)$. Some $h$ mutations lead to the formation of microchaetes in regions of the fly that are normally void of them, such as wing and the scutellum. The analysis of flies with different doses of $h$ and AS-C has suggested that $h$ directly controls AS-C expression, possibly by repressing achaete expression in defined regions (Sturtevant 1970; Moscoso del Prado and Garcia-Bellido 1984).

Although the case of $h$ demonstrates that segmentation genes can indeed be involved in the patterning of adult sense organs, it tells us nothing about the identity of the activator genes that were hypothesized above. It is possible that other segmentation genes or combinations thereof are involved in the regional activation of AS-C . A tantalizing example is provided by the gene hedgehog, which is expressed in the scutellar region of the notum and the postvertical region of the head (Móhler and Wieschaus 1985): this pattern of expression corresponds exactly to what one would expect for an activator acting at the $\mathrm{H}$ site. In an attempt to see if this correlation is more than a coincidence, N. Hansen in our laboratory 
made flies doubly heterozygous for scute and for hedgehog. Both mutations are completely recessive, thus, the double heterozygote should be completely normal. Yet such flies often lack a scutellar bristle, supporting the idea that the two genes are interrelated functionally (Botas et al. 1982).

If this speculation on the control of AS-C by segmentation genes is along the right lines, then for the first time in the analysis of development there would be a good possibility of bridging the gap between DNA and a complex adult pattern, and reaching a molecular understanding of morphogenesis, thus fulfilling the dream of T.H. Morgan.

\section{New answers to old riddles}

The molecular dissection of AS-C provides molecular support to the claim that scute can be subdivided into several smaller regions, each responsible for the development of one or a few macrochaetes. Actually, the assignment of bristles to the 'subgenes' of 1930 is amazingly identical to that shown in Figure 1 (Dubinin 1932). Yet it is also true that, so far as adult bristles are concerned, scute and achaete are simple genes, the inactivation of which removes all bristles. We now see that this dilemma reflects the inherent duality of the eukaryotic genes--their molecular functions, encoded in the translated sequence, and their regulation, which involves a far larger segment of the chromosome. Both aspects are essential to what we might call the phenotypic function of the gene, i.e., its contribution to the normal phenotype of the animal. While the molecular function and regulation may be easier to analyze, it seems clear that it is the combination of the two that matters for the animal and will ultimately have to be understood.

Knowing more about this dual aspect of the phenotypic function helps us to understand complex loci. In the case of $A S-C$, at least, the complexity stems from the interspersion of coding sequences and control sites, such that different control sites act on the same gene, while at the same time one control site may act on different genes. Since some of these genes are very similar, the interspersion of coding sequences and control sites may result in two genes substituting for each other, or being expressed in complementary regions, depending on the particular battery of control sites that are activated (as is the case for achaete and scute in larva and adult). Furthermore, the same gene can define very different patterns. In the adult, scute is involved in the precise location of some macrochaetes, in the regular spacing of the abdominal bristles, and in the formation of clusters of campaniform sensilla in the wing. Interestingly, breakpoints that are considered as extreme scute alleles because they suppress most of the scute macrochaetes remove but a small subset of the scute campaniform sensilla (L. Leyns, pers. comm.), suggesting that the same gene depends on different control sites for the generation of different patterns.

Another AS-C riddle is the fact that the different macrochaetes can be ordered in a series such that scute mu- tations remove adjacent bristles in the series (Fig. 2). However, as noted repeatedly by previous workers, neither the seriation nor the particular set of bristles removed by a given scute allele makes much sense in terms of development, morphology, or anything one could think of. If the phenotype of a scute mutant depends on the set of control sites that happen to be inactivated or disconnected by that particular mutation, then the origin of the seriation lies within AS-C, and the failure to detect any developmental correlate to this series is easily explained.

Perhaps the most important outcome of the recent work on AS-C is that it offers suggestions on the oldest riddle of all, the issue of morphogenesis (or pattern formation in the modern parlance). The central puzzle of pattern formation is that tissues that appear rather homogeneous turn out, when they differentiate, to feature different structures and cell types ordered in reproducible arrays. This phenomenon seems to imply some sort of positional information such that a cell at a particular location knows what it should do. In the fly embryo, genetic and molecular analyses suggest that positional information may be encoded in the spatially restricted patterns of expression of a set of interacting genes, generically called segmentation genes (Gergen 1987; Scott and O'Farrell 1986; the fact that gene interactions can be translated into spatial patterns of expression obviously implies a lot of cross talk between neighbors about gene activity).

It seems entirely possible, and wholly in line with the interpretation of Ruiz-Gomez and Modolell, that segmentation genes or related genes play a similar role later in development for the generation of adult forms and patterns. This obviously would account for the prepattern that Stern postulated in the formation of the adult pattern of bristles (Stern 1954b). In fact, with this system the undifferentiated epithelium would be intrinsically prepatterned, ready to provide positional information for any gene that comes under the appropriate control. Such a system of positional information would endow a tissue with the two opposite properties that are so baffling in the study of development: it would be both mosaic (because the domain of expression of each activator gene will define an independent morphogenetic field) and regulative (because gene interactions coupled by cell interactions would tend to re-establish a complete pattern of gene expression, and therefore a complete pattern of spatial positions). Our use of 'mosaic' and 'regulative' may seem to differ from the one agreed upon by experimental embryologists, referring to the presence of localized cytoplasmic determinants in eggs or early embryonic stages. However, the difference fades if the cytoplasmic determinants are the products of specific regulatory genes, as could be the case of the segmentation genes in the fly.

As a final note, we should mention that other genes seem to be involved in the determination of adult bristles, for example, extra-macrochaetae (Moscoso del Prado and Garcia-Bellido 1984), which apparently interacts with AS-C; the so-called neurogenic loci (Leh- 
mann et al. 1983; Dietrich and Campos-Ortega 1984), which may be involved in lateral inhibition; and a number of less characterized genes (Lindsley and Grell 1968). Furthermore, other mechanisms undoubtedly play a role in the formation of the final pattern of bristles in the adult fly; for example, cell migration, alignment along the antero-posterior axis and lateral inhibition. The superposition of different mechanisms may explain why even in the absence of a control region in AS-C, the corresponding bristle occasionally may form (although in general it will be much less precisely located). It would also explain why the wild-type pattern is highly resistant to perturbations. However, even if AS-C is not the entire story, the perspectives it has opened so far are heartening news for the developmental geneticist.

\section{Acknowledgments}

Our debt to Antonio Garcia-Bellido is vast, and so is our gratitude. We are also grateful to Yuh Nung Jan and Lily Yeh Jan for innumerable discussions and many a pleasant day, as well as for introducing us to the art of the Great Oriental Compromise. A.G. is chercheur qualifie of the Fonds National de la Recherche Scientifique (Belgium).

\section{References}

Agol, I.J. 1931. Step allelomorphism in Drosophila melanogaster. Genetics 16: 254-266.

Bate, C.M. 1978. Development of sensory systems in arthropods. In Handbook of sensory physiology (ed. M. Jacobson) vol. 9, pp. 1-53. Springer Verlag, Berlin, New York.

Botas, J., J. Moscoso del Prado, and A. Garcia-Bellido. 1982. Gene-dose titration analysis in the search of trans-regulatory genes in Drosophila. EMBO I. 1: 307-310.

Cabrera, C.V., A. Martinez-Arias, and M. Bate. 1987. The expression of three members of the achaete-scute complex correlates with neuroblast segregation in Drosophila. Cell 50: $425-433$.

Campos-Ortega, J.A. and V. Hartenstein. 1985. The embryonic development of Drosophila melanogaster. Springer Verlag, Berlin.

Campuzano, S., L. Carramolino, C.V. Cabrera, M. Ruiz-Gomez, R. Villares, A. Boronat, and J. Modolell. 1985. Molecular genetics of the achaete-scute gene complex of Drosophila melanogaster. Cell 40: 327-338.

Campuzano, S., L. Balcells, R. Villares, L. Carramolino, L. Garcia Alonso, and J. Modolell. 1986. Excess of function Hairy-wing mutations caused by gypsy and copia transposable elements inserted within structural genes of the achaete-scute locus of Drosophila. Cell 44: 303-312.

Carramolino, L., M. Ruiz-Gomez, M.C. Guerrero, S. Campuzano, and J. Modolell. 1982. DNA map of mutations at the scute locus of Drosophila melanogaster. EMBO I. 1: 11851191.

Child, G. 1935. Phenogenetic studies on scute-1 of Drosophila melanogaster. Genetics 20: 109-126.

Dambly-Chaudière, C. and A. Ghysen. 1986. The sense organs in the Drosophila larva and their relation to the embryonic pattern of sensory neurons. Wilhelm Roux's Arch. Dev. Biol. 195: 222-228.

1987. Independent subpatterns of sense organs require independent genes of the achaete-scute complex in Drosophila larvae. Genes Dev. 1: 1297-1306.

Dietrich, U. and J.A. Campos-Ortega. 1984. The expression of neurogenic loci in imaginal epidermal cells of Drosophila melanogaster. J. Neurogen. 1: 315-332.

Dubinin, N.P. 1932. Step-allelomorphism and the theory of centres of the gene, achaete-scute. J. Genet. 26: 37-58.

-1933. Step-allelomorphism in Drosophila melanogaster J. Genet. 27: 443-464.

Garcia Alonso, L. and A. Garcia-Bellido. 1986. Genetic analysis of Hairy-wing mutations. Wilhelm Roux's Arch. Dev. Biol. 195: $259-264$.

Garcia-Bellido, A. 1979. Genetic analysis of the achaete-scute system of Drosophila melanogaster. Genetics 91: 491-520.

1981. From the gene to the pattern: achaete differentiation. In Cellular controls in differentiation (ed. C.W. Lloyd and D.A. Rees/ pp. 281-304. Academic Press, London.

Garcia-Bellido, A. and J.R. Merriam. 1971. Parameters of the wing imaginal disc development of Drosophila melanogaster. Dev. Biol. 24: 61-87.

Garcia-Bellido, A. and P. Santamaria. 1978. Development analysis of the achaete-scute system of Drosophila melanogaster. Genetics 88: 469-486.

Gergen, J.P. 1987. Drosophila segmentation genes and blastoderm cell identities. BioEssays 6: 61-66.

Ghysen, A. 1980. The projection of sensory neurons in the central nervous system of Drosophila: Choice of the appropriate pathway. Dev. Biol. 78: 521-541.

Ghysen, A. and J. Richelle. 1979. Determination of sensory bristles and pattern formation in Drosophila. Dev. Biol. 70: $438-452$.

Goldschmidt, R.B. 1931. Die entwicklungsphysiologische Erklarung des Falls der sogennanten Treppenallelomorphe des genes scute von Drosophila. Biol. Zbl. 51: 508-526.

Goldschmidt, R.B. 1938. Physiological genetics. McGraw-Hill, New York and London.

Lehmann, R., F. Jimenez, U. Dietrich, and J. Campos-Ortega. 1983. On the phenotype and development of mutants of early neurogenesis in Drosophila melanogaster. Wilhelm Roux's Arch. Dev. Biol. 192: 62-74.

Lindsley, D.L. and E.H. Grell. 1968. Genetic variations of Drosophila melanogaster. Carnegie Institution of Washington Publication No. 627.

Mohler, N.N. and E. Wieschaus. 1985. Postembryonic requirements of the hedgehog gene of Drosophila melanogaster. Genetics 110 (suppl.): 35.

Moscoso del Prado, J. and A. Garcia-Bellido. 1984. Genetic regulation of the achaete-scute complex of Drosophila melanogaster. Wilhelm Roux's Arch. Dev. Biol. 193: 242-245.

Müller, H.J. and A.A. Prokofyeva. 1935. The individual gene in relation to the chromomere and the chromosome. Proc. Natl. Acad. Sci. 21: 16-26.

Nüsslein-Volhard, C. and E. Wieschaus. 1980. Mutation affecting segment number and polarity in Drosophila. Nature 287: 795-801.

Perrimon, N. and A.P. Mahowald. 1987. Multiple functions of segment polarity gene in Drosophila. Dev. Biol. 119: 587600.

Raffel, D. and H.J. Müller. 1940. Position effect and gene divisibility considered in connection with three strikingly similar scute mutations. Genetics 25: 541-582.

Romani, S., S. Campuzano, and J. Modolell. 1987. The achaetescute complex is expressed in neurogenic regions of Drosophila embryos. EMBO f. 6: 2085-2092.

Ruiz-Gomez, M. and J. Modolell. 1987. Deletion analysis of the 
achaete-scute locus of D. melanogaster. Genes Dev. 1: 1238-1246.

Scott, M.P. and P.H. O'Farrell. 1986. Spatial programming of gene expression in early Drosophila embryogenesis. Annu. Rev. Cell. Biol. 2: 49-80.

Serebrovsky, A.S. and N.P. Dubinin. 1930. X-ray experiments with Drosophila. I. Hered. 26: 469-478.

Stern, C. 1954a. Two or three bristles. Am. Sci. 42: 213-247.

1954b. Genes and developmental patterns. Caryologia (suppl.) 355-369.

1956. The genetic control of developmental competence and morphogenetic tissue interactions in genetic mosaics. Arch. Entwicklungsmech. Organ. 149: 1-25.

Sturtevant, A.H. 1970. Studies on the bristle pattern of Drosophila. Dev. Biol. 21: 48-61.

Sturtevant, A.H. and J. Schultz. 1931. The inadequacy of the sub-gene hypothesis of the nature of the scute allelomorphs of Drosophila. Proc. Nat1. Acad. Sci. 17: 265-270.

Villares, R. and C.V. Cabrera. 1987. The achaete-scute gene complex of $D$. melanogaster: Conserved domains in a subset of genes required for neurogenesis and their homology to myc. Cell 50: 415-424.

Wigglesworth, V.B. 1940. Local and general factors in the development of 'pattern' in Rhodnius prolixus. I. Exp. Biol. 17: $180-200$.

Wilkins, A. 1987. Neuroblast formation and the role of the AS-C gene complex in Drosophila. BioEssays 7: 82-84. 


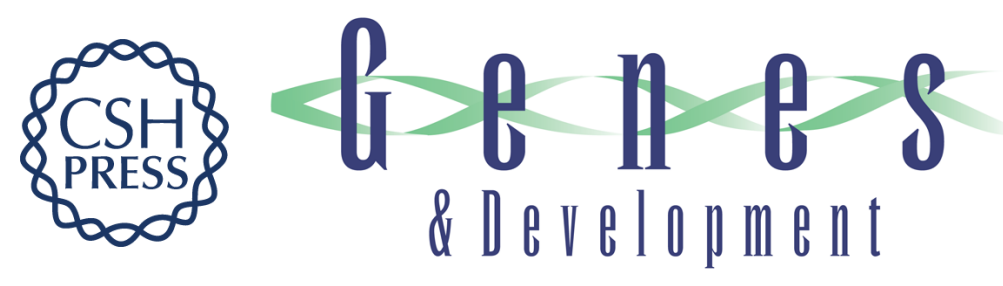

\section{From DNA to form: the achaete-scute complex.}

A Ghysen and C Dambly-Chaudière

Genes Dev. 1988, 2:

Access the most recent version at doi:10.1101/gad.2.5.495 $\begin{array}{ll}\text { References } & \begin{array}{l}\text { This article cites } 38 \text { articles, } 9 \text { of which can be accessed free at: } \\ \text { http://genesdev.cshlp.org/content/2/5/495.full.html\#ref-list-1 }\end{array}\end{array}$

\section{License}

Email Alerting

Receive free email alerts when new articles cite this article - sign up in the box at the top Service right corner of the article or click here.

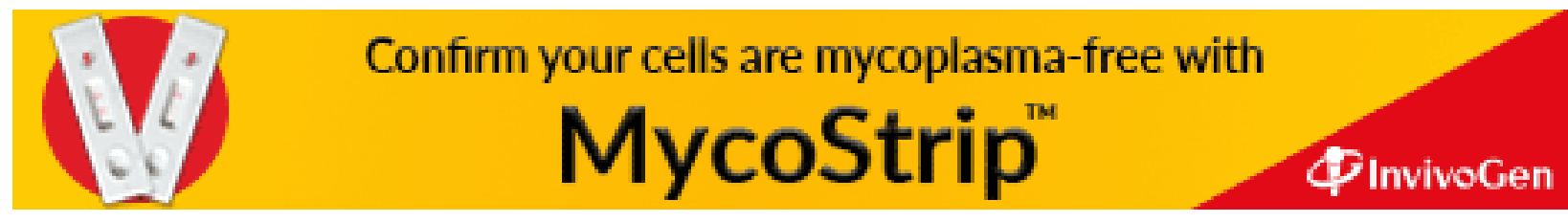

